\title{
Prácticas de responsabilidad social empresarial en la Pyme Un estudio en el sector metalmecánico de Barquisimeto, Estado Lara, Venezuela
}

\section{Zahira Moreno \\ Freites \\ Universidad Centroccidental Lisandro Alvarado, Venezuela}

\section{Dennys Graterol} Universidad Centroccidental Lisandro Alvarado rteacs@ucla.edu.ve

\section{Resumen}

Este estudio tiene como propósito realizar una investigación sobre las prácticas de Responsabilidad Social Empresarial (RSE) que lleva a cabo la pequeña y mediana empresa (Pyme) del sector metalmecánico en la Zona Industrial II de Barquisimeto, Estado Lara, Venezuela. La muestra estuvo conformada por siete empresas, a las cuales se les aplicó un cuestionario y una entrevista a profundidad. Se determinó la existencia de capacidades y recursos en la Pyme sin que éstos sean usados estratégicamente para fortalecer sus ventajas competitivas; también se evidenció la práctica de acciones comunes de RSE, toda vez que se considera importante la protección del ambiente, la reducción de energía, manejo de desechos, aguas residuales. No obstante, en aspectos relacionados con actuaciones en la comunidad, el mercado (proveedor-cliente) y el ambiente de los trabajadores no son abordados considerando los beneficios en términos de creación, fortalecimiento o mejoras de las capacidades competitivas de las empresas. Por lo tanto, se concluye que la Pyme del sector presenta un bajo nivel de apropiación de la práctica de RSE.

Palabras clave: Responsabilidad Social Empresarial, capacidades y recursos, ventajas competitivas, Pyme. 


\title{
Corporate Social Responsibility (CSR) in the SMEs' sector in Barquisimeto, Venezuela
}

\begin{abstract}
The present study intends to realize an investigation on Corporate Social Responsibility (CSR) as a management strategy in the metalworking sector of SMEs in the Industrial Area II of Barquisimeto - Lara State/Venezuela. The sample consisted of 7 SMEs which responded to a questionnaire and an in-depth interview. We discover the existence of capabilities and resources in the SMEs, which are not strategically used in order to strengthen their competitive advantages. The study showed the use of common actions of CSR as to the importance granted to environment protection, energy reduction, waste and wastewater management. However, issues related to actions in community, market (supplier-customer) and workers' mood are not equally addressed considering the benefits in terms of creating, strengthening or improving the competitive capabilities of these enterprises. It is therefore concluded that SMEs in the sector have a low degree of usage of CSR practices.
\end{abstract}

Keywords: Corporate Social Responsibility, capabilities and resources, competitive advantage, SMEs.

\section{Introducción}

La Responsabilidad Social Empresarial (RSE) es, de acuerdo con García y Zabala (2009: 115), "un instrumento corporativo que implica un compromiso de las empresas a través de la aplicación sistemática de recursos para respetar y promover los derechos de las personas, el crecimiento de la sociedad y el cuidado del ambiente".

Al respecto, Marín (2007, p. 245) resalta que "la importancia de utilizar teorías de identidad social, significa hasta qué punto las personas se identifican con la empresa"; sin embargo, la RSE puede definirse como un contrato social, tal como lo señala Bouckaert (1998: 216), indicando que "es una obligación de la organización motivada a las consecuencias originadas de sus acciones".

Así, pues, la responsabilidad que tienen las empresas ante los impactos de sus decisiones y actividades que ocasionan en la sociedad y el medio ambiente deben ser expresadas en un comportamiento organizacional que tome en consideración las expectativas de sus partes interesadas o stakeholders, que contribuya al desarrollo 
sostenible, la salud y el bienestar de la sociedad, que cumpla con la legislación vigente y sobre todo que esté integrada en toda la organización y forme parte de su identidad, cultura y valores.

Dado que las Pyme son empresas que representan una base importante de la economía, la RSE, —como estrategia empresarial y herramienta de gestión— es también aplicable a la Pyme, independiente del sector al que pertenezca; por lo tanto, la Pyme juega un papel importante en el desarrollo de la RSE.

En este contexto, Murillo y Lozano (2006), Torres (2007), Peinado (2007), Páez (2007) señalan las iniciativas y practicas sobre RSE que puede introducir la Pyme, pero depende de la naturaleza de cada una de ellas y de su entorno, para implementar la responsabilidad empresarial. En Venezuela, al igual que en el resto de América Latina, la mayor parte del sector productivo está conformado por la Pyme; en este sentido, existe una significativa participación de estas empresas del sector metalmecánico ubicadas en la Zona Industrial II de Barquisimeto, Estado Lara, donde fueron consultados los gerentes, administradores y directivos de siete empresas en relación con lo que entienden y hacen sobre RSE, lo cual ha permitido obtener información primaria con respecto a las capacidades y recursos de las empresas, así como a la orientación de los elementos claves para la RSE con el objetivo de identificar la potencialidad existente para la puesta en práctica de la RSE, una medida que fortalece la ventaja competitiva de la empresa.

$\mathrm{Al}$ respecto, la gerencia de las Pyme consultadas, en una primera aproximación para este estudio, reconoce la importancia de establecer la RSE en sus organizaciones debido a la necesidad que hoy en día tiene su compenetración con sus principales grupos de interés o stakeholder, recurriendo a mecanismos asociativos y de redes. Entre estos mecanismos se encuentran los gremios y cámaras empresariales, iniciativas sectoriales, entre otros (Páez, 2007, p. 19). Esta respuesta para muchos es vista como una presión del estado venezolano ante los cambios que se vienen introduciendo en el sector productivo del país.

De igual manera informaron que mantienen asignaciones presupuestarias para programas que, según ellos, tienen carácter de RSE. Éstos están dirigidos a formación del personal, seguridad e higiene laboral y preservación del medio ambiente; en consecuencia del establecimiento de un marco normativo legal, en el que se inscribe la Ley Orgánica de Prevención, Condiciones y Medio Ambiente de Trabajo LOPCYMAT, y la Ley Orgánica del Ambiente LOA. Por lo tanto, se trata más de 
una respuesta a cambios en el entorno legal que una decisión propia de asumir las responsabilidades con su entorno social. RSE

Según Páez (2007, p. 26) en la Pyme no se cuenta con una estrategia formalmente definida de RSE; asimismo, no se contempla una unidad orgánica, ni tampoco una persona responsable, que impulse la planificación de la RSE como componente gerencial; lo cual, conlleva a que los planes en relación con el desarrollo de alguna actividad relacionada con RSE se lleve a efecto sin la debida planificación en concordancia con los objetivos de rentabilidad de las Pymes y su visión estratégica de largo plazo.

En este orden de ideas, Páez (2007) y Moreno (2006) consideran importante establecer evaluaciones que permitan determinar el impacto de los programas de RSE debido a que no se tienen establecidos sistemas de control e indicadores que permitan evaluar los cambios en relación con la eficiencia de dichos programas o acciones. También reconocen la existencia de personal profesional de alto nivel, lo cual ha sido considerado una fortaleza para el establecimiento de la RSE como una estrategia gerencial para impulsar mejoras en los niveles competitivos de las empresas.

Las acciones que en esta área desarrolla la Pyme venezolana obedece a la necesidad de adherirse a lo establecido en la normativa que rige en el ámbito laboral respecto a los beneficios contractuales de los trabajadores y planes de formación, adiestramiento y desarrollo (Páez, 2007, p. 26), a lo que algunas de las fuentes consultadas han estimado como un logro de los trabajadores a partir de sus propias exigencias y no a partir, de acuerdo con Barroso (2007, p. 49), de una manera de "contribuir a la calidad de vida de la gente dentro de la organización [...] esto es, invertir en el personal y propiciar un ambiente de trabajo basado en el respeto a la dignidad del trabajador".

De acuerdo con lo señalado por Vallverdú (2003, p. 1), en relación con la acción responsable de las organizaciones hacia el colectivo que conforma su contexto, refiere la interrelación existente y necesaria entre empresa y sociedad a partir del principio de la responsabilidad social inherente a las organizaciones al expresar que "ser socialmente responsable no significa solamente cumplir plenamente las obligaciones jurídicas, sino ir más allá de su cumplimiento invirtiendo más en el capital humano, el entorno y las relaciones con los interlocutores". No obstante la realidad de la Pyme venezolana conlleva a un cumplimiento de las normativas le- 
gales como estrategia reactiva y adaptativa ante las presiones que experimentan en un ambiente altamente dinámico con presiones políticas, sociales y económicas.

Es así como, a razón de los argumentos planteados, surgen las siguientes interrogantes, en un contexto de alta presión y dinamismo como el venezolano: ¿la Pyme realmente utiliza sus capacidades y recursos para promover sus ventajas competitivas sobre la base de las políticas de RSE? o ¿la Pyme solamente alcanza a ser uso de sus capacidades y recursos para el cumplimiento de la normativa legal que se exige en el contexto social y político del país?

Las respuestas a estas cuestiones no son simples, la realidad de cada Pyme es compleja y particular. Sin embargo, resulta interesante abordar en esta investigación las prácticas de Responsabilidad Social Empresarial que se llevan a cabo en la Pyme del sector metalmecánico en la Zona Industrial II de Barquisimeto, Estado Lara, Venezuela. Para ello se efectuó lo siguiente: 1) identificar las capacidades y recursos en la Pyme; 2) diagnosticar las políticas de Responsabilidad Social Empresarial como estrategia gerencial por parte de la Pyme de este sector y 3) examinar la estrategia gerencial de la Pyme sobre la base de las políticas de Responsabilidad Social Empresarial diagnosticadas.

\section{Antecedentes}

En el estudio sobre la "Situación de la Responsabilidad de la Empresa en la Pyme de América Latina y el Caribe" (Peinado, 2007, p. 19) se revela que una gran mayoría de la Pyme latinoamericana realiza acciones y actividades ligadas con la RSE, a pesar de su desconocimiento formal del concepto en muchos casos. Atendiendo los resultados de este estudio, 6 de cada 10 microempresas realiza acciones relacionadas con RSE en un grado bajo y 3 de cada 10 en grado medio.

La publicación del Instituto ETHOS y el CEBRAE (2007), "Responsabilidad Social para Micro y Pequeñas Empresas - Paso a Paso" menciona que aquellas empresas que adoptan la filosofía y prácticas de la RSE tienden a tener una gestión más consciente y de mayor claridad en cuanto a su misión. Poseen un mayor compromiso por parte de los empleados, mejor relación con los proveedores y clientes, además de una imagen positiva ante la comunidad.

En el año 2007, y con el apoyo de la organización AVINA, se presentaron los informes de resultados de los trabajos de investigación sobre la cobertura de la RSE 
en los medios de prensa de 8 países: Argentina, Chile, Paraguay, Bolivia, Perú, Ecuador, España y Portugal. En el informe se presentaron varios casos que hacen referencia a las acciones emprendidas por la Pyme de los países mencionados, donde la mención de la RSE se destaca en sólo 3.5\% - 5.7 \% de la Pyme.

\section{Políticas de Responsabilidad Social Empresarial}

El desarrollo sostenible posee una dimensión económica, medioambiental y social, además es un objetivo global de la Unión Europea, por lo que la Dirección General de Empresa e Industria (DGEI) se propone crear las condiciones en las que las empresas europeas puedan prosperar, ayudándoles así a maximizar su contribución al desarrollo sostenible.

Planteamiento que se ha derivado en una serie de programas de concientización y formación para los empresarios europeos. De esta manera son notorios los esfuerzos que viene realizando la comisión europea en su programa Empresa Sostenible y Responsable (ESR), pues considera la responsabilidad social de las empresas como una acción necesaria y natural en el afán de lograr una mayor competitividad; por ello, resulta crucial que las empresas se comporten de manera responsable para generar confianza en la economía de mercado.

A continuación se describen políticas (DGEI-ESR) que permiten la implementación de la RSE en empresas pequeñas, medianas o grandes. Dejando de lado el prejuicio que existe de que la responsabilidad social sólo puede ser llevada adelante por grandes corporaciones y no por la Pyme. En este estudio queda claro lo que es RSE sin llegar a confundirla con acciones filantrópicas o de inversión social como lo hacen las grandes empresas con la creación de fundaciones, por ejemplo, situación por la que suele asociarse la RSE a decisiones y acciones que demandan grandes cantidades de dinero. La Pyme, por lo general, no suele tener excedentes económicos y tiempo suficiente para acciones sociales que la disperse de su propósito principal.

Es así que para este estudio de la Pyme metalmecánica de Barquisimeto Estado Lara,Venezuela, se aborda la RSE desde la perspectiva de las políticas que se presentan a continuación (DGEI-ESR): políticas de actuación en el lugar de trabajo, política de medio ambiente, política de mercado, política social y valor de la empresa. 


\section{Política de actuación en el lugar de trabajo}

Las empresas deben tener el respeto y consideración por la legislación relativa a los temas de contratación, seguridad y salud en el puesto de trabajo; puede bastar para satisfacer las necesidades básicas de sus trabajadores, un compromiso visible con la mejora de sus condiciones de trabajo, con su desarrollo profesional y con su bienestar personal, lo que demostrará que realmente son valorados como personas y que se considera su contribución al negocio. Involucrar a los empleados permitirá obtener algo más de ellos en forma de ideas, compromiso y lealtad, lo que redundará en una mayor eficiencia para la empresa.

Gracias a la importancia que se le da a los empleados, éstos se sienten identificados con la organización con sentido de pertenencia; entienden los valores de su empresa y su forma de trabajo, permitiéndoles así que aporten sus ideas, lo que ayudará a gestionar mejor los cambios que se introduzcan en la empresa.

Las políticas en el lugar de trabajo son un mecanismo para establecer una forma ordenada y disciplinada de operar en el lugar de trabajo, es más que la confianza y buenas relaciones internas entre el gerente dueño de la organización y sus trabajadores. Es importante considerar a los empleados como el factor primordial en el desempeño empresarial.

Hoy más que en ningún otro momento, los empleados constituyen el activo más importe de las empresas, considerándolos una fuente de ventaja competitiva. Según Devenport (2000, p. 22) "Los trabajadores son activos" se ha convertido en la metáfora dominante de los directivos de finales del siglo XX. En ciertos aspectos representa un ascenso apreciable de los empleados hacia el rango que merecen. Al fin y al cabo, las personas constituyen para la mayoría de las empresas el motor principal de su prosperidad".

\section{Política medioambiental}

Actualmente, en gran parte de los países, incluyendo Venezuela, existen graves problemas energéticos, por lo que el ahorro de energía se ha vuelto importante. De igual forma, la prevención de la contaminación, la reducción de residuos y el reciclaje permiten a la empresa reducir sus gastos, así como cumplir la normativa medioambiental, mejorar las relaciones con la comunidad local y fidelizar a sus 
empleados y tener lealtad de sus clientes. Todos estos beneficios contribuyen a la sostenibilidad de su empresa y su éxito a largo plazo. Según López et al., (2006, p. 125) "[...] las medidas de protección y cuidado ambiental no sólo ahorran costos sino son rentables, debemos aplicar las 3R: Reducir, Reciclar y Reutilizar"

Todas las empresas, independientemente de su tamaño o sector de actividad, pueden ejercer impactos positivos o negativos sobre el medio ambiente. Los impactos negativos proceden del consumo directo o indirecto de energía y recursos, la generación de residuos y agentes contaminantes y de la destrucción de los hábitats naturales.

Aunque las pequeñas empresas tienen menos impacto negativo medioambiental todas pueden ayudar reduciendo el consumo de energía, los residuos y reciclando materiales. Hasta los más discretos avances pueden significar una gran diferencia cuando se suman a los esfuerzos de otros negocios.

De aquí la importancia de poseer una política medioambiental, aun cuando está normalizado en las leyes debe formar parte de las estrategias empresariales en una organización netamente responsable con sus empleados, comunidades aledañas, proveedores, consumidores, clientes, entre otros.

\section{Política de mercado}

Las empresas son básicamente organizaciones humanas que dependen de una red de relaciones internas y externas vitales para la prosperidad de todos. Las buenas relaciones con los clientes y proveedores proporcionan beneficios a ambas partes. Una buena interacción es fundamental para lograr el éxito. Una forma rápida de mejorar la productividad de la empresa es compartir experiencias con los proveedores, clientes, empresas de ideas afines y organizaciones empresariales locales, además de otros grupos de interés para poder iniciar la implementación de la RSE en la cadena de valor.

Para conservar el buen nombre de la empresa, es importante "actuar correctamente" en todas sus relaciones laborales. En la práctica se puede lograr una buena reputación dejando satisfechos a los clientes, liquidando las facturas a tiempo y cumpliendo con todas las prácticas empresariales de forma ética. El buen nombre es crucial para asegurar el éxito en el mercado y ser sostenible en el tiempo. 


\section{Política social}

Muchos de los pequeños negocios son vitales para la comunidad local y tienen una activa implicación en las aspiraciones y actividades del lugar. Dichas empresas se benefician de cosas como las siguientes:

- Unas valiosas relaciones personales y vínculos con otras empresas de la zona.

- Una mayor aceptación y estima con el cliente.

- Una mejor reputación empresarial.

- Una mejor contratación y retención del personal.

El apoyo a la comunidad puede incluir cualquier actividad que beneficie a la colectividad, como el patrocinio o la dedicación de tiempo y experiencia; además, puede incluir la participación en iniciativas culturales, educativas o deportivas; ayudar a solucionar cuestiones sociales, como la prevención del delito o el desempleo a largo plazo en su área también puede contribuir al éxito económico duradero de su negocio.

Asimismo, participar activamente en la comunidad también puede ayudar a identificar nuevos mercados, clientes u oportunidades de negocio; establecer contactos con las autoridades locales y líderes de opinión, y facilitar nuevas asociaciones con otros negocios en proyectos comunitarios. Es importante realizar aquellas actividades que respondan a los objetivos de la empresa (actividades que interesen a los posibles clientes o destinadas a satisfacer las necesidades de formación y empleo de su empresa), que sean relevantes para su comunidad, que animen a sus empleados a participar y que estén al alcance de las posibilidades de la empresa.

Es importante implementar una estrategia a la medida de los recursos que la empresa disponga, pero es fundamental adoptar una "causa social" que esté ligada a la estrategia de negocio para que la empresa aporte su experiencia y conocimiento a la causa social que ha decidido apoyar.

\section{Valores de la empresa}

Las pequeñas y medianas empresas con frecuencia reflejan los valores y principios del dueño/director. Sin embargo, algunas empresas han plasmado sus valores en un código de ética o de conducta, que se traducen en una declaración de buenas 
prácticas empresariales o incluso en una simple serie de normas que articulan la visión, los valores, las responsabilidades y ambiciones de la empresa.

Al respecto, Fernández (2006) señala que una actuación ética no debe reducirse a una especie de código que diga qué debo hacer o no, sino que se debe comprender las implicaciones que conlleva el actuar éticamente en toda acción humana. La ética no es un añadido a la acción directiva, es esencial. En este mismo orden de ideas, el autor indica que para un empresario, los convenios morales al tomar decisiones son fundamentalmente los mismos que rigen a las demás actividades humanas, precisamente porque el director es un ser humano.

Es por ello que al actuar de manera ética se ofrecen unas directrices coherentes sobre cómo afrontar las situaciones que provocan problemas o conflictos de intereses. Los resultados serán mejores cuando el empresario convenza a los demás mediante su propia forma de conducirse, de su compromiso con los valores declarados por su empresa, expresados o no de manera explícita, pero procurando que estén indisolublemente ligados con normas éticas y morales con una reputación positiva ante la comunidad. En consecuencia, al tener unos valores fortalecidos, y las políticas de actuación en el lugar de trabajo, política de medio ambiente, política de mercado y política social, la empresa se convierte en una organización socialmente responsable con gran prestigio y altamente valorizada, situación que le genera una ventaja competitiva en el mercado y la convierte en un modelo a imitar por sus competidores.

\section{Diseño metodológico}

La población se encuentra constituida por quince pequeñas y medianas empresas del sector metalmecánico ubicadas en la Zona Industrial II de Barquisimeto en el estado Lara. La muestra se ha seleccionado de acuerdo con un muestreo intencionado o sesgado que, según Hurtado y Toro (1997, p. 45), es aquel en el cual "la muestra no se elige al azar, sino que por razones determinadas, el investigador decide quiénes serán los integrantes de la misma"; en este sentido, el investigador ha seleccionado aquellas empresas del sector que han manifestado interés en la práctica de RSE; por lo tanto, en reunión previa con la Cámara de Artesanos, Pequeños y Medianos Industriales del Estado Lara (CAPMIL), la muestra seleccionada quedó conformada por siete empresas: Metalúrgicas Caruso e Hijos C.A Andamios Dalmine C.A; Techo Duro C.A; Fundiciones Metalúrgicas Lemos C.A; Hidrojet C.A; Domínguez \& cía., S.A; Ingeniería Industrial Metalmecánica S.A. 
Se contactó vía telefónica las citas para la aplicación del cuestionario y entrevista (mayo, 2009); después se realizaron dos sesiones de trabajo, durante los meses junio y julio 2009 con los gerentes de administración, gerentes de recursos humanos, gerentes de planta y en algunos casos con el dueño o gerente general de la Pyme. Se utilizó la técnica de la entrevista en profundidad; para su aplicación se hizo uso de un instrumento de recolección de información bajo la modalidad de guión estructurado a fin de direccionar las entrevistas. Con este fin la entrevista busca conocer el nivel de conocimiento que tienen los entrevistados sobre la definición y conceptualización de la RSE, las preguntas fueron formuladas para investigar qué entienden los entrevistados por RSE y así establecer un criterio único a la hora de responder y posteriormente analizar los resultados.

También se utilizó el cuestionario como instrumento para la recolección de datos "Cuestionario de Concienciación - Empresa Responsable" desarrollado por el Programa Empresa Sostenible y Responsable (DGEI-ESR) de la Dirección General de Empresa e Industria de la Comunidad Europea. Este instrumento está estructurado en 26 preguntas, con 5 alternativas de respuesta en la escala de Liker, que corresponden a las siguientes políticas organizacionales: política de actuación en el lugar de trabajo, política de medio ambiente, política de mercado, política social y valor de la empresa.

\section{Discusión de los resultados}

Explorar la comprensión que los gerentes y empresarios tienen acerca de la RSE ayuda a entender sus prácticas. Por ello, se iniciaron las entrevistas con la pregunta sobre el concepto de RSE para tener una idea del nivel de conciencia que tienen de las dimensiones de la RSE (trabajadores, comunidad y medio ambiente) y de las políticas que coadyuvan a la implementación de la RSE (políticas de actuación en el lugar de trabajo, política de medio ambiente, política de mercado, política social y valor de la empresa), lo cual está expresado en el cuestionario que posteriormente contestarían.

Después de efectuar lo anterior, se encontró que la gran mayoría de los entrevistados no tiene claro lo que significa RSE. Creen que está destinado únicamente a las grandes empresas. Que para ellos es actuar en favor de la sociedad sin obtener ningún beneficio empresarial a cambio. Cuando se les explicó que la RSE consiste básicamente en conservar la rentabilidad de la empresa y obtener una ventaja 
competitiva creándose así una buena reputación y ganándose la confianza de las personas que trabajan para la empresa o viven cerca de ella, entonces sus comentarios se orientaron hacia el cumplimiento de las leyes. Desde este contexto, se hace evidente que la mayoría asocia la RSE con el cuidado del ambiente, la capacitación y seguridad de los trabajadores, sobre todo cuando el gobierno actual ha enfatizado la fiscalización para el cumplimiento de estas leyes. También consideran importante tener buenas relaciones con las comunidades locales ya que la figura de comuna que maneja el gobierno da mayor poder a los consejos comunales y estos ejercen gran influencia en las empresas.

Esta manera de concebir la RSE es comprensible debido a las presiones que ejerce el gobierno actual en el sector productivo venezolano, donde toda iniciativa gubernamental es vista como amenaza y peligro para la propiedad privada. Es en este periodo gubernamental donde más se observa el estímulo (voluntario y coercitivo) para el desarrollo de la RSE. Se trata de integrar las políticas empresariales de RSE con las políticas gubernamentales, pues para la responsabilidad social, según el gobierno, no sólo debe limitarse a aspectos voluntarios, sino que se está trabajando en las regulaciones para que sea puesta en práctica por las empresas.

En cuanto a las respuestas que dieron los consultados al cuestionario, para ampliar y profundizar los aspectos en que se ha dimensionado la RSE para este estudio, los resultados son los siguientes:

Variable: capacidades y recursos.

Dimensión: capacidades y recursos físicos, financieros, humanos, tecnológicos, organizacionales y de reputación.

De acuerdo con la información aportada por las fuentes consultadas y mediante el apoyo de la entrevista estructurada se observó que la Pyme posee una serie de capacidades y recursos que no se encuentran integrados a una estrategia gerencial alrededor de la RSE para el fortalecimiento de sus ventajas competitivas. No obstante, se puede deducir que las iniciativas de RSE que adelantan son de carácter reactiva y adaptativa: "reacciona ante las demandas y solicitudes de trabajadores y comunidades; bajo la fuerte presión que ejerce el marco legal venezolano". El cumplimiento de las leyes y de las normas mínimas de conducta que se imponen en el país estarían entonces coadyuvando a cimentar las bases para un desarrollo social responsable y sostenido en estas empresas. 


\section{Cuadro 1}

\section{Capacidades y recursos}

\begin{tabular}{|l|l|r|}
\hline \multirow{2}{*}{ Descripción de capacidad - recurso } & \multicolumn{2}{|c|}{ EXISTENTE } \\
\cline { 2 - 3 } & SÍ & NO \\
\hline
\end{tabular}

\begin{tabular}{|l|l|l|}
\hline Recursos: & & \\
\hline Físicos & & \\
\hline Instalaciones para formación y adiestramiento & $\mathrm{X}$ & \\
\hline Financieros & & \\
\hline Recursos monetarios para disponer en RSE & & $\mathrm{X}$ \\
\hline Acceso a financiamientos & $\mathrm{X}$ & \\
\hline Humanos & & \\
\hline Personal profesional y capacitado en RSE & $\mathrm{X}$ & \\
\hline Tecnológicos & & \\
\hline Sistemas de información & $\mathrm{X}$ & \\
\hline Procesos de mejora continua & $\mathrm{X}$ & \\
\hline Normas de calidad & $\mathrm{X}$ & \\
\hline Organizacionales & & \\
\hline Unidad orgánica y/o persona responsable de la RSE & & $\mathrm{X}$ \\
\hline Gerencias funcionales & $\mathrm{X}$ & \\
\hline Reputación & & \\
\hline Atención al cliente & $\mathrm{X}$ & \\
\hline Sistema de proveedores & $\mathrm{X}$ & \\
\hline Imagen corporativa & $\mathrm{X}$ & \\
\hline Productos de calidad & $\mathrm{X}$ & \\
\hline Capacidades: & & \\
\hline Planificación y Administración por objetivos de RSE & & $\mathrm{X}$ \\
\hline Motivación para emprender acciones RSE & & $\mathrm{X}$ \\
\hline Información sobre RSE y visión de largo plazo & $\mathrm{X}$ & $\mathrm{X}$ \\
\hline Relaciones con las comunidades & $\mathrm{X}$ & \\
\hline Conocimiento sobre RSE & $\mathrm{X}$ & \\
\hline Percepción filantrópica de RSE & & $\mathrm{X}$ \\
\hline Práctica de RSE de acuerdo con leyes & & \\
\hline Percepción de RSE como elemento competitivo & & \\
\hline & & \\
\hline & & \\
\hline & & \\
\hline & & \\
\hline & & \\
\hline & & \\
\hline & & \\
\hline & & \\
\hline
\end{tabular}

De acuerdo con los resultados que se reflejan en el cuadro 1 las capacidades de la Pyme en general, se pudieran englobar en conocimientos, habilidades, actitudes y 
experiencia del personal con respecto a la RSE; incluye también el conocimiento sistematizado hecho explícito por la organización, específicamente en procesos de trabajo, técnicas, rutinas o programas de RSE. Al respecto la evidencia empírica demuestra que las Pyme consultadas no disponen de información y la motivación no es suficiente para emprender con fuerza programas y acciones de RSE que va sumado a la falta de planificación y estrategia de RSE (Páez 2007, p. 26). Asimismo, se encuentra el problema de los recursos económicos que son limitados; además, no se visualiza el beneficio de la RSE a largo plazo porque sus necesidades y su esfuerzo para mantenerse en el mercado toman toda su atención.

Relacionado a los recursos, en el cuadro 1 también se evidencia la escasa capacidad técnica de su personal para las acciones comunitarias. Igualmente, se resalta la poca disponibilidad de tiempo para dedicarse a labores de RSE debido a la gran cantidad de funciones que se cumplen al interior de la Pyme donde las prioridades son otras, siendo la responsabilidad social un labor adicional.

Variable: políticas de Responsabilidad Social Empresarial.

Dimensiones: política de actuación en el lugar de trabajo, política medioambiental, política de mercado, política social y valores de la empresa.

\section{Cuadro 2}

\section{Política de Responsabilidad Social Empresarial}

\begin{tabular}{|c|c|}
\hline \multicolumn{2}{|c|}{ Política de Responsabilidad Social Empresarial } \\
\hline Instrumento de recolección & Cuestionario \\
\hline
\end{tabular}

\begin{tabular}{|l|c|c|}
\hline \multirow{2}{*}{\multicolumn{1}{|c|}{ Participación de Política RSE }} & \multicolumn{2}{|c|}{ ítems: 1 al 26 } \\
\cline { 2 - 3 } & Número & \% \\
\hline Política de actuación en el lugar de trabajo & 35 & $17 \%$ \\
\hline Política Medioambiental & 58 & $29 \%$ \\
\hline Política de Mercado & 41 & $20 \%$ \\
\hline Política Social & 36 & $18 \%$ \\
\hline Valores de la empresa & 33 & $16 \%$ \\
\hline
\end{tabular}

Fuente: Elaboración propia a partir del estudio de campo. 


\section{Gráfico 1 \\ Política de Responsabilidad Social Empresarial}

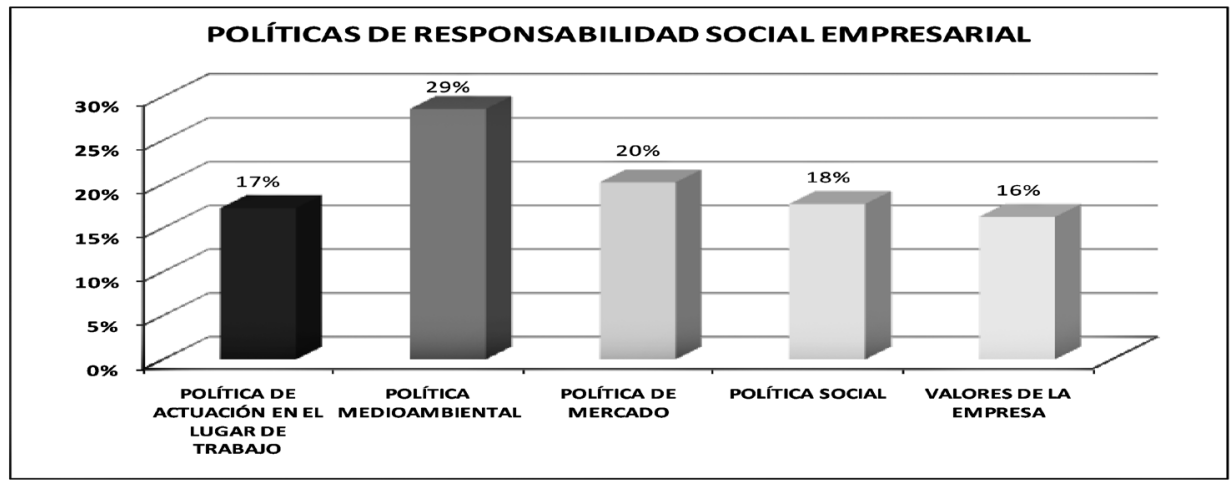

Fuente: Elaboración propia a partir del estudio de campo.

Según el cuadro 2, la evidencia empírica muestra que las actividades o programas que lleva la Pyme sobre RSE en primer lugar de importancia se encuentran las orientadas a las actividades ambientales con $29 \%$, luego sobresale su énfasis en el mercado y relaciones con los clientes y proveedores con $20 \%$. No menos importante para ellos es la formación de los trabajadores y acciones dirigidas al apoyo de las comunidades con $18 \%$ y en menor proporción se realizan acciones orientadas hacia la mejora del clima y cultura en el ambiente de trabajo y el fortalecimiento de valores sociales con $16 \%$.

De acuerdo con estos resultados se evidencia una alta tendencia hacia satisfacer los requerimientos de protección y preservación del medio ambiente. Esta actuación por parte de la Pyme se encuentra fundamentada en la existencia de leyes y normas de obligatoria observancia. Es común observar en la mayoría de las Pyme estudiadas, programas de protección ambiental, manejo de residuos y desechos, con la convicción de que los mismos son necesarios para la preservación de los ambientes naturales y espacios para futuras generaciones además de contribuir directamente con la disminución del impacto de riesgos ambientales que ocasionan sus operaciones (Páez 2007, p. 25).

De igual forma, la Pyme se encuentra orientada en forma favorable a la implementación de políticas dirigidas hacia el mercado de su interés, las cuales se encuentran 
orientados por los objetivos de rentabilidad; esto establece su interés funcional y operacional en relación con mantener la eficiencia en sus operaciones. Además, señalan que en cuanto a las relaciones con sus proveedores y clientes éstas son muy buenas y cordiales, situación que los hace responsables con los compromisos adquiridos. No obstante, no se evidenció claramente alguna acción relacionada específicamente con la RSE; sin embargo, afirman que los productos que compran a sus proveedores cumplen con los estándares de calidad que ellos necesitan para ofrecer a sus clientes el mejor servicio/producto, considerándolo como una acción ética para competir en el mercado, situación que consideran de mucha importancia y requiere de mucho esfuerzo por parte de ellos.

Seguidamente, la orientación de la Pyme se encuentra hacia la implementación de políticas sociales con el objeto de mantener la inserción laboral y de la organización en su contexto. Con respecto a su integración con la comunidad, se encontró que los trabajadores son colaboradores activos en el patrocinio de eventos deportivos, además de contribuir con ciertos proyectos que demandan los consejos comunales. Algunos entrevistados manifestaron el poder que tienen las comunidades a través de las leyes que se han venido aprobando en el país; por lo tanto, hay que considerarlos como un actor importante en la gestión de la empresa.

La actuación en el lugar de trabajo y valores de la empresa son las acciones que presentan la menor atención de la Pyme sin reparar en el hecho de que a partir de éstas se fortalecen las competencias y capacidades de las organizaciones. A pesar de ello, la muestra consultada ha expuesto que se mantienen programas de formación y fortalecimiento al personal de sus organizaciones. Además de cumplir con todo lo que exige la ley para los trabajadores.

Variable: estrategia gerencial.

Dimensión: gerencia estratégica.

De acuerdo con lo expresado por las fuentes consultadas, las acciones o actividades por parte de la Pyme pudiesen ser ligeramente caracterizadas en el marco de una política de Responsabilidad Social Empresarial a pesar de que su mayoría se encuentra orientada en función de las leyes establecidas vinculadas o vinculantes a la preservación del medio ambiente, manejo de residuos o desechos, la observación de normas de seguridad industrial e higiene, entre otras. 
La estrategia empresarial, que se observa en esta práctica de RSE, es la de atender el marco regulatorio en áreas específicas, y no la de propiciar ambientes para la efectiva planificación de la Responsabilidad Social orientada en concordancia con los objetivos corporativos de rentabilidad y sostenibilidad. Por lo tanto, sus estrategias son reactivas y adaptativas en función de cómo el entorno regulatorio va imponiendo nuevas leyes (ley federal de los consejos comunales, ley orgánica del ambiente, ley de protección e higiene laboral, entre otras de reciente data para los empresarios venezolanos) la Pyme va reaccionando con acciones puntuales para no ser sancionadas. Adatándose progresivamente a estas exigencias, por lo que aún no se podría hablar explícitamente de RSE en este tipo de empresas porque que debe ser asumida como una serie completa de políticas, prácticas y programas que están integrados en todas las operaciones y políticas de la Pyme.

No obstante, el cumplimiento de las leyes y de las normas mínimas de conducta que se imponen en el país nos permite cimentar las bases para un desarrollo social responsable y sostenido en estas Pyme.

\section{Conclusiones}

Los resultados obtenidos en esta investigación permiten tener una visión aproximada de la RSE, en el grupo de las Pyme consultadas al momento de realizar el presente estudio (junio-julio 2009). Por lo tanto, se concluye que actualmente las acciones emprendidas por la Pyme no descansan sobre la base de la construcción de ventajas competitivas a partir del uso eficiente de sus capacidades y recursos. Estas empresas poseen capacidades y recursos que no son administrados en forma estratégica; cuentan con recursos financieros, tecnológicos, físicos, humanos, organizacionales y de reputación sin que éstos se encuentren integrados a los objetivos corporativos de rentabilidad; por lo tanto, carecen de una visión de largo plazo y una planificación estratégica que incluya las dimensiones de la RSE de manera sostenible en el tiempo.

La existencia de las capacidades y recursos mencionados, a pesar de sus limitaciones, plantea un escenario positivo para la puesta en práctica de la RSE; en consecuencia, se recomienda establecer lineamientos estratégicos con el fin de orientar la implantación de la RSE en las Pyme del sector metalmecánico ubicadas en la Zona Industrial II de Barquisimeto sobre la base de los recursos y capacidades existentes en estas empresas para su desarrollo, administración y control. Para ello, 
la Pyme debe considerar la RSE de manera integrada con la estrategia empresarial, la cual debe ser claramente definida y coherente con el negocio y construida a partir de la participación de los diferentes grupos de interés de estas empresas.

Las Pyme consultadas reflejan elementos comunes en materia de Responsabilidad Social Empresarial, toda vez que consideran importante la protección del ambiente, la reducción de energía, residuos y aguas residuales; también se encuentran definidas, aunque con menor intensidad, en cuanto al rol de la identidad corporativa de sus valores, así como las condiciones del ambiente de trabajo.

En consecuencia, presentan un bajo nivel de apropiación en la práctica de los elementos establecidos en la Responsabilidad Social Empresarial; esto atenta al enriquecimiento del capital social de las pequeñas y medianas empresas y se constituye en un factor que afecta la gestión empresarial para el fortalecimiento de las capacidades y ventajas competitivas de sus organizaciones en una sociedad que demanda su participación activa en los problemas de su comunidad vecina.

Las acciones que actualmente emprenden en materia de RSE se encuentran únicamente supeditadas al estricto cumplimiento de normas establecidas en diferentes áreas y que afectan su contexto. De ahí la importancia de integrar la RSE al modelo de gestión que llevan la pequeña y mediana empresa a través de una buen proceso de planificación estratégica.

\section{Bibliografía}

Barroso, F. (2007). Responsabilidad Social Empresarial y sugerencias para su aplicación en instituciones educativas. Caracas: Universidad Simón Bolívar.

Bouckeaert, L. (1998). Management of non-profit institutions. Journal of Business Ethics.

Devenport, T. (2000). Capital humano. Creando ventaja competitiva a través de las personas. Madrid: Gestión 2000.

ETHOS y CEBRAE (2007). Empresas \& Impresa: Pauta de responsabilida de Brasil. Ponencia, Primer Foro de Responsabilidad Social Empresarial del MERCOSUR. 
Fernández B. (2006). Ética de la dirección: decisión con libertad. México: Ediciones Ruz.

Fundación AVINA (2007). Responsabilidad Social Empresarial en la prensa paraguaya. Un análisis de la cobertura periodística sobre la RSE. Asunción. Ponencia, Primer Foro de Responsabilidad Social Empresarial del MERCOSUR.

Fundación para el Desarrollo de la zona Centro Occidental (2002). Dossier estado Lara. Venezuela.

García. J. y H. Zabala (2008). Políticas de Estado como sustento de la organización ciudadana. Centro de Investigación de Ciencias Administrativas y Gerenciales. Volumen 5, Edición 1. Universidad Belloso Chacín.

Herrera E (2007). El valor de la reputación corporativa en la organización del siglo XXI. Disponible en: http://www.reddircom.org/textos/valorreputacion. pdf (consulta: febrero 22, 2009)

Hurtado y Toro (1997). Paradigmas y métodos de investigación en tiempos de cambio. Episteme

Marín, L. (2007). ; I need you too! Corporat identiy atractiveness for consumers and the role of Social Responsibility. Journal of Buiness Ethics. Tomo 71 No. 3.

Moreno, A. (2006). Percepción y práctica de los líderes de Pymes de Caracas con respecto a la Responsabilidad Social Empresarial. Trabajo de grado Msc. de la Universidad Católica Andrés bello. Facultad de Ciencias Económicas y Sociales. Escuela de Ciencias Sociales.

Murillo, D. y J. Lozano (2006). RSE y PYMES: Una apuesta por la excelencia empresarial. Instituto Persona, Empresa y Sociedad IPES.

López I., F. Chagollán y J. Martín del Campo (2006). Ecología. México: Umbral. 
Páez. T. (2007). Teoría y práctica de la ética y la Responsabilidad Social de la Pyme Venezolana. Revista FACES Universidad de Carabobo. Volumen XVIII No. 2: 165-183.

Peinado, Vara (2007). La responsabilidad social y medioambiental de la microempresa en latinoamérica. Primer Foro de Responsabilidad Social Empresarial del MERCOSUR

Tarrés E. (2007). ¿Es posible la Responsabilidad Social en la Pyme? Revista Tribuna de Opinión No. 7.

Vallverdú, J. (2003). Papeles de ética, economía y dirección. La responsabilidad social de la empresa: una aportación contable. Departamento de Contabilidad. Facultad de Ciencias Económicas y Empresariales. Universidad de Barcelona. $N^{o}$ 8. España. Disponible en: http://www.eben-spain.org/docs/ Papeles/XI/2_Valleverdu.pdf (consulta: Mayo 17, 2010). 